\title{
RIESGO DE SUICIDIO EN JÓVENES UNIVERSITARIOS DE BUCARAMANGA EN EL AÑO 2011
}

\author{
SUICIDE RISK IN COLLEGE STUDENTS FROM BUCARAMANGA IN THE YEAR 2011 \\ Eliana Martínez-Duran ${ }^{2}$, Marcela Romero-Romero ${ }^{2}$, Nelly Rey de Cruz $z^{3}$, Wilson Cañón-Montañez ${ }^{4}$
}

\section{RESUMEN}

Introducción: El suicidio es un fenómeno social con una alta tasa de mortalidad entre los jóvenes que pasan por varias etapas de cambios físicos y emocionales, con los intentos o pensamientos de suicidio común sentir miedo de lo que podría suceder en una situación difícil por la que están pasando. Santander en Colombia y las mayores tasas de suicidio son los jóvenes y la tendencia a aumentar. Todavía no sabemos la magnitud del problema en este grupo de alto riesgo, sin llegar a un suicidio total. Por lo tanto, esta investigación busca comprender este problema de una manera real de los que están vivos y en riesgo de suicidio y no esperar hasta que se trate y/o morir por esta causa Materiales y Métodos: Estudio descriptivo, observacional de corte transversal, en una muestra de 343 estudiantes de la Facultad de Ingenierías de la Universidad de Santander de Bucaramanga; mediante una encuesta utilizando la Escala de Riesgo suicida Plutchik y otras variables socio demografías conformada por 27 preguntas. Se validan en dos bases de datos del programa EPIDATA, posteriormente se exportan al programa STATA 10, para el análisis estadístico. Resultados: El $64.1 \%$ de los participantes fueron hombres, $35.9 \%$ son mujeres; prevaleciendo las edades $18(15.7 \%)$ y $20(14.6 \%)$; el $11.7 \%$ tenían antecedentes personales de suicidio; la ingeniería con mayor riesgo suicida fueron Civil 13\%, Electrónica $6.2 \%$, Ambiental 4.9\%, Industrial 3.6\%; evidenciando en los factores socio demográficos que solo el $83.4 \%$ gozan de una buena funcionalidad familiar; $51.3 \%$ consume alcohol y el 2.3\% depende de éste. Discusión y Conclusiones: Los jóvenes son más vulnerables a tener riesgo suicidio debido a los múltiples factores socio demográficos: disfunción familiar, antecedentes de suicidio y consumo de alcohol, cigarrillo, sustancias psicoactivas. (Rev Cuid 2011; 2(2): 182-87)

Palabras clave: Suicidio, Ideación Suicida, Jóvenes, Factores de Riesgo. (Fuente: DeCS BIREME).

\section{ABSTRACT}

Introduction: Suicide is a social phenomenon with a high mortality rate among young people who go through multiple stages of physical and emotional changes, with attempts or suicidal thoughts common to feel fear of what might happen in a difficult situation by that are happening. Santander in Colombia and the highest rates of suicide are young people and the tendency to increase. Still do not know the magnitude of the problem in this high risk group, without reaching a complete suicide. Therefore, this research seeks to understand this problem in a real way of those who are alive and at risk of suicide and not wait until they try it and / or die for this cause. Materials and Methods: A descriptive, observational cross-sectional in a sample of 343 students of the Faculty of Engineering at the University of Santander in Bucaramanga, a survey using the Plutchik Suicide Risk Scale and other socio demographics consists of 27 questions . Validated in two databases EPIDATA then exported to STATA 10 for statistical analysis. Results: $64.1 \%$ of participants were men, $35.9 \%$ are women ages 18 to prevail $(15.7 \%)$ and $20(14.6 \%)$, $11.7 \%$ had a history of suicide, the more suicidality Facultad of Engineering Civil were 13\% Electronics 6.2\% Environmental $4.9 \% 3.6 \%$ Industrial, evidenced in the socio-demographic factors that only $83.4 \%$ have a good family functioning, $51.3 \%$ consumed alcohol and 2.3\% dependent on this. Discussion and Conclusions: Young people are more vulnerable to having suicidal risk because of multiple socio demographic factors, family dysfunction, history of suicide and alcohol consumption, smoking, psychoactive substances.

Key words: Suicide, Suicidal Ideation, Youth, Risk Factors. (Source: DeCS BIREME).

\footnotetext{
${ }^{1}$ Artículo Original de Investigación.

${ }^{2}$ Estudiantes de Enfermería de Noveno Semestre, Universidad de Santander, Bucaramanga-Colombia.

${ }^{3}$ Enfermera, Especialista en Salud Mental. Docente, Programa de Enfermería, Universidad de Santander UDES.

${ }^{4}$ Enfermero. MSc en Epidemiología. Profesor de Planta. Programa de Enfermería. Grupo de Investigación de Enfermería-Everest (Categoría B Colciencias). Universidad de Santander UDES. Bucaramanga. Santander. Colombia.

Autor para Correspondencia: Marcela Romero Romero, E-Mail: marchs189@hotmail.com. Articulo recibido el 5 de Septiembre y aceptado para publicación el 20 de Octubre de 2011.
} 


\section{INTRODUCCIÓN}

El suicidio, constituye un evento multicausal, con reconocidas implicaciones personales, familiares, sociales y económicas, de alta complejidad. La ideación suicida impacta cada vez más en la población joven entre 18 a 25 años de edad y según datos de la "Organización Mundial de la Salud, este es el segmento poblacional con mayor riesgo suicida y con tendencia a incrementarse más rápido en todo el mundo"(1).

Se han identificado algunos factores que inciden sobre este evento con mayor frecuencia en los jóvenes, como es la depresión, la cual se considera uno de los factores que más se ha vinculado con el suicidio. Dicho trastorno afecta de manera importante el nivel de funcionamiento personal y social (2).

Es por esto que a lo largo de la historia se han elaborado diversos conceptos y descripciones de este fenómeno, llegando al consenso de que el suicidio es una conducta compleja en la que intervienen factores de todo tipo como son los socios demográficos, psicológicos y biológicos. Debido a esto se ha visto una gran incrementación de la tasa de suicidio en la población adolescente de, Finlandia Canadá y Argentina (3).

Otro de los factores que aumenta el riesgo de la conducta suicida es el consumo de sustancias psicoactivas, pues su contacto con el organismo puede modificar tanto las funciones orgánicas como los estados de conciencia, el estado de ánimo y los procesos de pensamiento (4).

En Colombia el suicidio es considerado como un problema de salud pública. De acuerdo con estadísticas del Instituto de Medicina Legal y Ciencias Forenses (INMLF), el País presenta una tasa de $7.4 \%$ suicidios por cada 100 mil habitantes por año, principalmente entre personas de 18 a 25 años de edad (5).

El departamento de Santander ha ocupado uno de los 10 primeros puestos con esta problemática, y al igual que en el país, las más altas tasas de suicidio la ocupan los jóvenes. Por esto es importante que los jóvenes con ideación y conducta suicida, reciban una atención profesional integral por parte del equipo de salud mental: Psiquiatra, Psicologo y la Enfemera Especialista en Salud Mental, para así evitar de una forma u otra que sigan aumentando estas tasas; ya que nuestro país no cuenta con información epidemiológica sistemática sobre trastornos mentales o conflictos psicológicos entre los jóvenes, ni mucho menos con la infraestructura y recursos necesarios para su atención (6).

El proceso dinámico y complejo del suicidio pasa por varias etapas antes de culminar en el acto que le quita la vida a la persona. Sus fases previas pueden ser identificadas oportunamente para dar pie a la intervención adecuada. De este modo, el conocimiento específico de la dinámica del suicidio sumado al reconocimiento de los factores de riesgo asociado a la ideación y comportamiento suicida, podría reducir sus tasas, previniendo estos eventos y disminuyendo estas consecuencias causadas a nivel social y familiar (7).

El presente proyecto sobre el riesgo de suicidio en jóvenes de la universidad de Santander de Bucaramanga se realiza con el fin de evaluar problemática de una manera real, reconociendo su relevancia en éste grupo poblacional tan afectado.

\section{MATERIALES Y MÉTODOS}

Tipo de diseño metodológico: Estudio descriptivo, observacional de corte transversal.

Población de estudio: $(\mathrm{N}=753)$ estudiantes de pregrado de la Universidad de Santander, modalidad presencial, matriculados en el calendario A del año 2011, de ambos sexos, diferentes edades, todos los estratos socioeconómicos y diferentes sitios de procedencia, del programa de Ingeniería (industrial, civil, sistemas, alimentos, electrónica y ambiental).

Prueba Piloto: La prueba piloto, se realizo con el $10 \%$ del tamaño muestra el cual fue de 36 estudiantes de Enfermería de la UDES, matriculados en el semestre A 2011, los cuales se distribuyeron por semestre (1 semestre:5; 2 semestre:4; 3 semestre:4; 4 semestre:2; 5 semestre:2; 6 semestre:3; 7 semestre:3; 8 semestre:4; 9 semestre:5; 10 semestre:4) lo que queríamos lograr con la prueba piloto, es saber si las preguntas realizadas en las encuestas, son las correctas y si nos arrojan los resultados esperados, verificar su eficiencia para así poder aplicar el instrumento a la población objeto de estudio.

En la prueba piloto se utilizo una encuesta para conocer las variables socio demográficas de la población objeto de estudio. El instrumento utilizado estaba conformado por 42 preguntas distribuidas así, (27 preguntas de información sobre las características socio demográficas y otras de interés. Se aplico además la escala de riesgo suicida de Plutchik (Risk of Suicide, RS) la cual discrimina entre las personas con y sin riesgo suicida.

Esta escala consta de 15 preguntas dicotómicas. Según los autores cuanto mayor sea la puntuación mayor es el riesgo.

Con la prueba piloto pudimos notar la necesidad de algunos cambios en las preguntas socio demográficas y otras de interés para mayor entendimiento de los estu- 
diantes en el momento de responderlas y se dejo la variable edad de forma abierta para poder calcular en que rango de edades se encontraba mayor riesgo suicida.

Tamaño de muestra: Conformada por 343 estudiantes de ingenierías distribuido de manera proporcional así: ING. industrial: 108; ING. Civil: 134; ING. Sistemas: 8; ING. Alimentos: 1; ING. Electrónica: 31; ING. Ambiental: 61

Tipo de muestreo: No probabilístico por conveniencia.

Periodo del estudio: Primer periodo académico del A 2011

Instrumento para la recolección de datos: Se utilizó la Escala de Riesgo Suicida de Plutchik (Risk of Suicide, RS) (8). En su versión española. Esta escala autoaplicada consta de 15 ítems con respuesta dicotómica (si/ no) que incluyen cuestiones relacionadas con intentos autolíticos previos, intensidad de la ideación actual, sentimientos de depresión, desesperanza y otros aspectos relacionados con las tentativas. Cada respuesta afirmativa puntúa 1 punto y cada respuesta negativa 0 puntos, con un rango de posibles puntuaciones totales entre $0 \mathrm{y}$ 15. Según los autores cuanto mayor sea la puntuación mayor es el riesgo.

Para este estudio se utilizaron otras variables socio demográficas de interés las cuales nos ayudan a mirar la población de forma global para obtener un mejor análisis del estudio. Dentro de las que se encuentran la edad, sexo, procedencia, religión, estrato socioeconómico relaciones familiares, núcleo familiar, rendimiento académico, entre otras.

Procedimientos: La prueba piloto se aplico a 36 estudiantes de Enfermería de la Universidad de Santander UDES (que corresponden al 10\% del tamaño muestra) con el fin de observar el comportamiento de la escala de Riesgo Suicida de Plutchik, y de otras variables socio demográficas. Los estudiantes se seleccionaron de una forma aleatoria.

Realizado los análisis correspondientes de la prueba piloto, este estudio se continúo con la población de muestreo, estudiantes de los diferentes Programas de las Ingenierías.

Se realizaron los cambios correspondientes en el instrumento que se aplicó definitivamente.

Hecha la convocatoria, los investigadores procedieron a la sensibilización (explicación breve sobre la naturaleza, objetivos, métodos, relevancia, riesgos y beneficios del estudio). Los instrumentos se administraron colec- tivamente en el horario habitual de clases y fueron entregados a cada estudiante en la siguiente secuencia: a) formato de consentimiento informado, b) Ficha sociodemográfica, y c) Escala de Riesgo Suicida de Plutchik. Para dar cumplimiento a las normas éticas respecto a la investigación con seres humanos, se diseñó y aplicó un formato para obtener el consentimiento informado como requisito autónomo para poder ejecutar este estudio.

Se aplicó una encuesta, por medio de la cual se registraron todas las variables sociodemográficas y de interés anteriormente mencionadas.

El punto de corte propuesto para la escala de Riesgo Suicida de Plutchik son 6 puntos, la cual nos muestra una fiabilidad de 0.90 , sensibilidad de $74 \%$ y una especificidad de $95 \%$ para discriminar entre individuos con antecedentes de tentativa de suicidio y sin antecedentes (9).

La sistematización de la información se realizó por medio del subprograma stat calc, a través del cual se hicieron la base de datos del estudio. Adicionalmente, se utilizó el paquete estadístico EPIINFO, versión 6.04 (10), con un nivel de confianza del $95 \%$, poder del $80 \%$ y una probabilidad de cometer error del $5 \%$.

La información recolectada se trascribió por separado en dos bases de datos en EPIDATA 3.1, la corrección de errores de trascripción se hizo mediante el subprograma Valídate, posteriormente las bases de datos se exportaron al programa EPIDATA ANALYSIS, para el análisis estadístico. Durante la entrada de datos se pueden realizar cálculos o controlar los valores que pueden introducirse. Se puede seleccionar un valor de una lista y guardar su código numérico $(1=\mathrm{No} 2=\mathrm{Si})$, la lista de textos se exportará con"etiquetas" para su uso en paquetes estadísticos.

Consideraciones éticas: Esta investigación ctuvo en cuenta en la Resolución 8430 de 1993 del Ministerio de la Protección Social de Colombia, la cual establece las normas científicas, técnicas y administrativas para la investigación en salud y por la ley 1090 del 2006 por la cual, se dicta el código deontológico y bioético y otras disposiciones.

Respeto y autonomía: La participación de los estudiantes en la investigación fue de manera libre. Para integrarse al estudio se solicitó el consentimiento informado escrito del estudiante.

Beneficencia - No Maleficencia: Esta investigación se clasificó como estudio "sin riesgo", según el numeral b del Artículo 11, de la Resolución 008430 del 04 de 
Octubre de 1993 del Ministerio de Salud de la República de Colombia. El diseño metodológico propuesto fue adecuado y los investigadores eran personas idóneas para realizar el estudio.

Justicia: Se incluyeron a estudiantes universitarios del Programa de ingenierías de la UDES matriculados para el primer periodo académico del año 2011 que aceptarón participar en la investigación. No se excluyó a ningún estudiante por razones de raza, género o credo religioso. Ningún estudiante implicado en el estudio, recibír patrocinios sociales, políticos, económicos o laborales, como retribución por su participación.

Confidencialidad: La información se conservo bajo estricta privacidad y solo será utilizable con fines académicos y científicos.

\section{RESULTADOS}

En este proyecto participaron 343 estudiantes de las diferentes Facultad de ingenierías (civil, industrial, alimentos, ambiental, sistemas y electrónica) de la Universidad de Santander matriculados en el periodo académico A del 2011; de los cuales el $15.7 \%$ y el $11.4 \%$ se encontraban entre las edades de 18 y 22 años, de los cuales $64.1 \%$ eran hombres y el $35.9 \%$ mujeres siendo estos la mayoría solteros con un $92.4 \%$, casados el $2.9 \%$ y el unión libre el $4.7 \%$,los ingresos económicos son considerados buenos en $72.9 \%$, regular $24.2 \%$ y no adecuado el $2.9 \%$, el $90.7 \%$ de los participantes no tienen hijos. En cuanto a la procedencia se puede ver un predominio del área urbana con un 95\%, encontrándose en el estrato tres con un $41.1 \%$ y cuatro un $30.6 \%$.

Respecto a la tipología familiar la mayoría de las familias son nucleares $67.9 \%$, en orden de frecuencia le sigue uniparental $13.7 \%$. En cuanto al número de integrantes no son familias muy numerosas, el $78.1 \%$ se halla entre 0 y 5 personas por grupo familiar y solo un $1.5 \%$ cuentan con más de 10 integrantes. Estas personas encuestadas algunos tienen hermanos, entre ellos ocupan un lugar el $61.8 \%$ está entre el 1y 2 y solo el $9.3 \%$ son hijos únicos; donde el $83.4 \%$ tienen una buena funcionalidad familiar, solo el $15.7 \%$ considera que es regular.

El $72.3 \%$ realizan actividades extra académicas. El deporte aparece en primer lugar de dichas actividades con un $93.2 \%$ de la población y el $60.6 \%$ realiza otras. En cuanto a la religión, el $85.1 \%$ afirma pertenecer a algún grupo religioso, católico en su mayoría $79.6 \%$.

El $11.7 \%$ presenta antecedentes personales de intento de suicidio. El $67.2 \%$, y antecedentes de abuso sexual el $1.7 \%$, respectivamente había asistido a consulta psicología y el $16.7 \%$ a consulta con el psiquiatra, el $2.6 \%$ manifestó tener una enfermedad crónica y el 1.2\% tienen un trastorno mental.

El $80.1 \%$ no fuman y $51.3 \%$ consume alcohol presentando una dependencia alcohólica $2.3 \%$, el $4.4 \%$ manifiesta consumir sustancias psicoactivas, ubicándose en primer lugar la marihuana con un $18.6 \%$ seguida por el bazuco, poppers y estaxis con un $1.6 \%$.

En la escala de riesgo suicida de Plutchick se encontró un promedio de riesgo del 2.27 con una desviación estándar del 2.7, En el total de la población, de los cuales solo 58 estudiantes presentan riesgo suicida esto equivalen a un $16.9 \%$. en esta escala también pudimos mirar el riesgo suicida por ingenierías teniendo en cuenta el punto de corte: 6 , y se observó en ingeniería civil un riesgo en el $13 \%$ y el $8.4 \%$ de los (130 estudiantes),en ingeniería ambiental $4.9 \%$ y $3.2 \%$ de (62 estudiantes), en ingeniería industrial $3.6 \%$ y $1.8 \%$ de (109 estudiantes), ingeniería electrónica $6.2 \%$ y $3.1 \%$ de(32 estudiantes) ; en las ingenierías de alimentos y sistemas no se presentó riesgo suicida (Ver Tabla 1). 
TABLA 1.Porcentajes de Riesgo Suicida por Facultades de Ingeniería

\begin{tabular}{|c|c|c|c|c|c|c|}
\hline $\begin{array}{c}\text { PUNTO DE } \\
\text { CORTE DE } \\
\text { LA ESCALA } \\
\text { DE PLUTCHICK }\end{array}$ & $\begin{array}{c}\text { ING. } \\
\text { CIVIL } \\
(\mathbf{n}=\mathbf{1 3 0})\end{array}$ & $\begin{array}{c}\text { ING. } \\
\text { AMBIENTAL } \\
(\mathbf{n = 6 2})\end{array}$ & $\begin{array}{c}\text { ING. } \\
\text { INDUSTRIAL } \\
(\mathbf{n = 1 0 9 )}\end{array}$ & $\begin{array}{c}\text { ING. } \\
\text { ALIMENTOS } \\
(\mathbf{n}=\mathbf{1})\end{array}$ & $\begin{array}{c}\text { ING. } \\
\text { SISTEMAS } \\
(\mathbf{n}=\mathbf{8})\end{array}$ & $\begin{array}{c}\text { ING. } \\
\text { ELECTRONICA } \\
(\mathbf{n}=\mathbf{3 2})\end{array}$ \\
\hline 6 & $8.46 \%$ & $3.2 \%$ & $3.6 \%$ & $0 \%$ & $0 \%$ & $3.1 \%$ \\
\hline 7 & $13 \%$ & $4.9 \%$ & $1.8 \%$ & $0 \%$ & $0 \%$ & $0 \%$ \\
\hline 8 & $2.3 \%$ & $1.7 \%$ & $1.8 \%$ & $0 \%$ & $0 \%$ & $6.2 \%$ \\
\hline 9 & $1.5 \%$ & $3.2 \%$ & $0 \%$ & $0 \%$ & $0 \%$ & $3.1 \%$ \\
\hline 10 & $0 \%$ & $3.2 \%$ & $1.8 \%$ & $0 \%$ & $0 \%$ & $0 \%$ \\
\hline 12 & $0.7 \%$ & $0 \%$ & $0 \%$ & $0 \%$ & $0 \%$ & $0 \%$ \\
\hline
\end{tabular}

\section{DISCUSIÓN}

En general los resultados de este proyecto nos confirman lo establecido en la literatura e investigaciones sobre el tema, como la edad en la que los jóvenes tienen mayor riesgo suicida son los que se encuentran entre los $18(15.7 \%)$ y $22(11.4 \%)$ años los cuales la gran mayoría son hombres con un (64.1\%) estos datos se puede relacionar con la crisis de la juventud, ya que es la etapa en que se concretan o no los proyectos y se presentan importantes cambios sociales en la vida profesional y familiar en quienes se reconoce que pasan por cambios físicos, psicológicos y emocionales que les hace vulnerables al suicidio, como una forma de escapar de sus conflictos internos y externos (6).

También podemos ver que el ingreso económico es regular con un $24.2 \%$ influye en el riesgo suicida como factor socio demográfico.

La procedencia en su mayoría corresponde al área urbana, la literatura revela el fenómeno del comportamiento suicida como un evento urbano.

La mayoría son solteros y no tenían hijos, lo cual se relaciona con los grupos de edades más afectadas y coincide con la literatura que muestra que las personas sin hijos y solteros tienen mayor predisposición al intento suicida ya que se encuentran solos y los sentimientos de tristeza los hacen más vulnerables.

\section{CONCLUSIONES}

Se puede concluir que la población joven, con determinadas características socio demográficas, muchas veces ve los problemas como algo irresoluble, haciendo así que el suicidio se convierte en la única alternativa.

Otro elemento a tomar en cuenta en este grupo de edad como es la adolescencia es el consumo de sustancias psicoactivas como el alcohol, que puede inducir a pensamientos y conductas suicidas, ya que perjudica la cognición y el humor, y disminuye la inhibición. Por sus efectos tóxicos, el alcohol también propicia sentimientos como la desesperanza y altera el desempeño de los neurotransmisores, los cuales son responsables del humor y del juicio. En estas circunstancias, el consumo de alcohol puede motivar en las jóvenes el rompimiento de relaciones interpersonales importantes y a la evasión de la ayuda social. De manera similar, otras sustancias como marihuana, cocaína y éxtasis traen consecuencias como el fracaso académico y ponen al adolescente en riesgo de accidentes, violencia y relaciones sexuales no planificado e inseguro y por ende llevarlos a tener pensamientos suicidas y a la realización de estos hechos.

\section{RECOMENDACIONES}

Los datos obtenidos y analizados en esta investigación evidencian la necesidad de invitar a los educadores para que se capaciten y se vinculen a un sistema de alerta epidemiológica que rápidamente permita identificar posibles factores de riesgo relacionados con la conducta suicida y remitan, si es el caso, a un profesional de la salud.

Se destaca la necesidad de realizar un acompañamiento cercano a los jóvenes e identificar los riesgos suicidas para intervenir oportunamente en ellos. 


\section{REFERENCIAS BIBLIOGRÁFICAS}

1. OMS (Organización Mundial de la Salud). El Informe Mundial de la Salud 2000. Salud mental: nuevos conocimientos, nuevas esperanzas, 2001a.

2. Leyva JR, Hernández JA, Nava JG, López GV. Depresión en adolescentes y funcionamiento familiar. Rev Med Inst Mex Seguro Soc 2007; 45(3): 225-232.

3. Wild L, Flisher A, Lombard C. Suicidal ideation and attempts in adolescents: associations with depression and six domains of self esteem. Journal of adolescence 2004; 27: 611-624.

4. Cardona AM, Hernández HF. Consumo de alcohol y otros psicoactivos en estudiantes universitarios y su relación con aspectos de la calidad de vida y factores psicosociales, Medellín 2003-2004.

5. Forensis. Centro de Referencia Nacional sobre Violencia. Instituto Nacional de Medicina Legal y Ciencias Forenses;2000. p.118-141.

6. Medina G. Itinerario con destino desconocido. Los jóvenes con rumbo al trabajo. Jóvenes, Cuarta Época 1997; 1(3):18-26.

7. Ana María Chávez-Hernández. Modelo psicoeducativo para la prevención del suicidio en jóvenes. Universidad de Guanajuato. 2008.

8. Plutchik R, Van Praga HM, Conte HR, Picard S. Correlates of suicide and violence risk: the suicide risk measure. Compr Psychiatry. 1989;30(4):296-302.

9. Rubio G, Montero I, Jáuregui J, Villanueva R, Casado MA, Marín JJ, et al. Validación de la escala de riesgo suicida de Plutchik en población española. Arch Neurobiol. 1998;61(2):143-52.

10. Centers for Diseases Control \& Prevention, Word Heath Organization. Version 6.04 Geneva: EPIINFO; 2000. 